\title{
Erratum to: Exponential Stability of Discrete-Time Delayed Hopfield Neural Networks with Stochastic Perturbations and Impulses
}

Sichao Wu and Chuandong Li

\section{Erratum to: Results Math \\ DOI 10.1007/s00025-011-0131-z}

It is the policy of the journal that the names of the authors appear in alphabetical order. As the authors of the above mentioned article disagree with this rule, they have made the following decision: the co-authors Shukai Duan and Wenfeng $\mathrm{Hu}$ have agreed that their names should be deleted from the list of authors. Sichao $\mathrm{Wu}$ and Chuandong $\mathrm{Li}$ are now the sole authors of the article "Exponential Stability of Discrete-Time Delayed Hopfield Neural Networks with Stochastic Perturbations and Impulses."

Sichao $\mathrm{Wu}$ and Chuandong Li

College of Computer

Chongqing University

Chongqing 400044

China

e-mail: wscfrank@sina.com;

licd@cqu.edu.cn

The online version of the original article can be found under doi:10.1007/s00025-011-0131-z. 